\title{
Optimization of Performance Evaluation of Urban Road Pavement
}

\author{
Ziyi Zhang ${ }^{1, \text { a }}$, Yu Sun ${ }^{1, b^{*}}$, Han $\mathrm{Xu}^{1}$, Qiyun $\mathrm{Zhu}^{1}$ \\ ${ }^{1}$ University of Shanghai for Science \& Technology, Department of Transportation Engineering, Yangpu, Shanghai, China
}

\begin{abstract}
In recent years, urbanization has developed rapidly, and urban road play a vital role as the premise. Due to the good effectiveness of asphalt pavement, which is more popular in urban road, and road maintenance demands are also increasing. In order to make the maintenance work appropriate, accurate pavement performance evaluation is the premise. This paper collects the data of a road pavement condition in Shanghai and calculates the sub-indexes of each section. We use the entropy weight method to obtain the influence degree of each sub-index. Then we use the revised set pair analysis to construct the comprehensive performance evaluation model of urban road pavement. The analysis shows that compared with the standard method and the set pair analysis, the revised model is more objective, in line with the actual use of the road.
\end{abstract}

\section{Introduction}

The construction and development of urban road have become a common concern. However, with the increase of urban road service life, the performance of the road gradually decreases because of the environment, vehicles, human factors and so on. Pavement performance's evaluation models are mostly highway while few studies on urban road, so it is important to study the pavement performance of urban road.

The concept of pavement performance was first proposed by AASHTO. Around the 1950s, the Road West Association summarized the Road Service Performance Index (PSI) based on road tests ${ }^{[1]}$. In many countries, based on the structure and parameters of PSI, the data and unified evaluation scale were linked to establish pavement performance models for various purposes, including RCI of Canada ${ }^{[2]}$ and MCI of Japan ${ }^{[3]}$.

In order to make the evaluation more objective and persuasive, many quantitative analytical studies have emerged. Majidifard, $\mathrm{H}$ et al. used the new machine learning technology to predict the rutting depth and improved the accuracy of the rutting curve ${ }^{[4]}$. Fan et al. established the evaluation method of pavement performance by considering five pavement performance indexes ${ }^{[5]}$. Yang SQ used the fuzzy evaluating method to provide a new theoretical method for accurate evaluation and analysis of highways pavement performance in semiarid climate zones ${ }^{[6]}$. Fan XY proposed an improved entropy weight-analytic hierarchy process to evaluate preventive pavement maintenance ${ }^{[7]}$. The evaluation models in recent years also include BP neural network, extension neural network, TOPSIS theory, PCA-SVM evaluation, improved grey clustering.

They understood the actual situation of pavement through single or comprehensive evaluation index, and provided scientific basis for later maintenance decisionmaking schemes and designs. The following problems can be found in the current pavement performance evaluation.

The research on the evaluation methods mainly focused on the pavement performance synthetically estimation. While the single evaluation index selection, evaluation method and the rationality analysis of single evaluation index was rarely involved.

The subjective factors of the evaluation model had great influence leading to the weak objectivity. The weight of evaluation indexes were fixed, which was not consistent with the actual condition of pavement.

In order to accurate predict the real situation of pavement performance; it is necessary to achieve accurate pavement performance evaluation from the aspects of data collection and method selection. By investigating the actual road conditions in Shanghai, this paper establishes an improved set pair analysis by using entropy weight method and team analysis method, and selects specific sections to verify the rationality of the method.

\section{Pavement Performance Modeling}

\subsection{Set Pair Analysis Theory}

\subsubsection{Applicability of Set Pair Analysis}

According to the current road data, we found that there is a contradiction in individual roads between the comprehensive evaluation and the sub-item evaluation results when using the specification for evaluation. For example, the A-level index of PCI in main roads is $[90,100]$. If the measured value on a certain road is 89 , the evaluation level is B and if the value is 91, the evaluation index is A. The objective fact that the identical

\footnotetext{
ayzhang918@163.com

* corresponding author: bysun@usst.edu.cn
} 
discrepancy contrary in each evaluation level has been ignored. The set pair analysis can well solve such problems.

The pavement performance evaluation based on set pair analysis is different from the standard method, which has dynamic characteristics. It can provide different assignment methods for $i$ and $j$ in the connection degree expression, which makes the research problems more indepth. Secondly, it can comprehensively evaluate the actual performance of pavement by using the relationship among identity, difference, opposition degree and the weight of above three indexes to evaluate the actual pavement grade.

The set pair analysis based on the existing evaluation indexes: road riding quality index (RQI) and pavement condition index (PCI). The PQI is set $\omega$ and the evaluation standard is set $\{\mathrm{A}, \mathrm{B}, \mathrm{C}, \mathrm{D}\}$. And then constitute four set pairs: $H(w, A), H(w, B), H(w, C), H(w, D)$. The corresponding contact degrees are $\mu(w-A), \mu(w-B)$, $\mu(w-C), \mu(w-D)$.

Due to the linear continuity of the evaluation function and the randomness of the road conditions of each section, the equidistant distribution of the difference coefficient is assigned according to the distance between the same degree and the opposite degree. The assignment of difference values corresponding to each set pair are shown in Table 1.

Table 1 Distribution of Evaluation Indexes and Grade Differences

\begin{tabular}{|c|c|c|c|c|}
\hline & $H(w, A)$ & $H(w, \mathrm{~B})$ & $H(w, \mathrm{C})$ & $H(w, \mathrm{D})$ \\
\hline$i_{A}$ & 1 & 0 & -1 & -1 \\
\hline$i_{B}$ & $1 / 3$ & 1 & 0 & $-1 / 3$ \\
\hline$i_{C}$ & $-1 / 3$ & 0 & 1 & $1 / 3$ \\
\hline$i_{D}$ & -1 & -1 & 0 & 1 \\
\hline
\end{tabular}

Pavement quality index PQI is set $\omega_{1}$, and then the calculation formulas of the connection degree of the four sets are:

$$
\begin{aligned}
& \mu\left(w_{1}-A\right)=v_{1} \times i_{A}+v_{2} \times i_{B}+v_{3} \times i_{c}+v_{4} \times i_{D} \\
& \mu\left(w_{1}-B\right)=v_{1} \times i_{A}+v_{2} \times i_{B}+v_{3} \times i_{c}+v_{4} \times i_{D} \\
& \mu\left(w_{1}-C\right)=v_{1} \times i_{A}+v_{2} \times i_{B}+v_{3} \times i_{c}+v_{4} \times i_{D} \\
& \mu\left(w_{1}-D\right)=v_{1} \times i_{A}+v_{2} \times i_{B}+v_{3} \times i_{c}+v_{4} \times i_{D}
\end{aligned}
$$

In the formulas, $v_{1} \sim v_{4}$ are weights of coefficient of variance, which is determined by the influence of each sub-index on PQI, and $v_{1}+v_{2}+v_{3}+v_{4}=1$. Since only RQI and PCI are the two influencing factors in the evaluation system, there are at least two indexes are not zero. Eventually the maximum value in $\mu(w-A), \mu(w-B)$, $\mu(w-C), \mu(w-D)$ is obtained, which is the final result of comprehensive evaluation in this section.

\subsubsection{Application of Revised Set Pair Analysis}

The above method is more comprehensive than the standard, but considering that each sub-index is within the same level range, the road conditions are quite different. When determining the values of identity, difference and opposition, revisions should also be made to the difference of scores. The revised $i$ value is shown in 5 .

$$
i_{X}^{\prime}=\left(1 \pm|| \frac{C_{R Q I}-C_{x}}{\Delta x}|-| \frac{C_{P C I}-C_{y}}{\Delta y} \|\right) \times i_{X}
$$

In the formula: $i_{x}$ is the value before revision, $i_{X}^{\prime}$ is the value after revision. $C_{R Q I}, C_{P C I}$ are the evaluation values of RQI and PCI; $\Delta x$ is the length of the rating interval at $C_{R Q I}$ and $\Delta y$ is the length of the rating interval at $C_{P C I} . C_{x}$ and $C_{y}$ is the upper limit or lower limit of the rating where $C_{R Q I} . C_{P C I}$ is located, and the lower limit is taken when $C_{R Q I}$ is above $C_{P C I}$, otherwise the upper limit is taken.

$$
\left|\frac{C_{R Q I}-C_{x}}{\Delta x}\right| \text { and }\left|\frac{C_{P C I}-C_{y}}{\Delta y}\right| \text { represent the deviation }
$$

degrees of the upper and lower limits about RQI and PCI compared with their respective evaluation levels. The higher the deviation percentage, the greater the impact of the sub-index on the PQI, and it takes positive if the index influence degree is bigger, otherwise takes negative.

Finally, the maximum value of $\mu(w-A), \mu(w-B)$, $\mu(w-C), \mu(w-D)$ is obtained, and the corresponding grade is the final result of the comprehensive evaluation of the section.

\subsection{Weight of Sub-Index Determined by Entropy Weight}

In order to obtain the evaluation level of road comprehensive performance more objectively and accurately, this paper selects the entropy weight method to assign the values of sub-index PCI and RQI, and studies the evaluation method of pavement performance from data. The basic calculation steps are as follows:

1) Data normalization

Due to the inconsistent unit system of the two sub-indexes, so the data need to be normalized. The normalization formulas of positive index and negative index are calculated as follows:

$$
X_{i j}=\left\{\begin{array}{l}
\frac{x_{i j}-\min \left(x_{j}\right)}{\max \left(x_{j}\right)-\min \left(x_{j}\right)} \\
\frac{\max \left(x_{j}\right)-x_{i j}}{\max \left(x_{j}\right)-\min \left(x_{j}\right)}
\end{array}\right.
$$

Where, $i$ is the number of measured sections, $i=1,2 \ldots n$; $j$ is the number of sub-index, $j=1,2 ; x_{i j}$ is the original data of index $j$ for section $i ; x_{i j}$ is the data after normalization.

2) Calculation of information entropy of each index

It shows that the greater the variability of indicators, the more information they provide.

$$
E_{j}=-\frac{1}{\ln (n)} \sum_{i=1}^{n} p_{i j} \ln \left(p_{i j}\right)
$$




$$
p_{i j}=\frac{X_{i j}}{\sum_{i=1}^{n} X_{i j}}
$$

Where, $E_{j}$ is the information entropy of index $j$; If $p_{i j}=0$, defining $\lim p_{i j} \ln \left(p_{i j}\right)=0$.

3) Determine the weight of indexes

The obtained information entropy calculates the weight of each index:

$$
v_{j}=\frac{1-E_{j}}{k-\sum E_{j}}
$$

Substituting the final weight of each sub-index into the set pair analysis and the revised set pair analysis, the comprehensive evaluation results of the above two methods can be obtained by calculation.

\section{Data Comparison and Analysis}

\subsection{Data Processing of Set Pair Analysis}

In this paper, we selected 85 sections of a municipal road in Shanghai, by calculating the indexes, the weight of RQI is 0.59 , while the weight of PCI is 0.41 . In order to further verify the reliability, the weight ratio is compared as "Technical Regulations for Shanghai Urban Road Maintenance"'(DG/TJ08-92-2013). We found that the weight ratio are similar to the calculated, proving the rationality and reliability of the entropy weight method.

In order to verify the effectiveness of the optimization method, we selected 20 typical sections from the calculation results, as shown in table 2 .

Table 2 Road grade obtained by three methods

\begin{tabular}{|c|c|c|c|c|c|c|c|}
\hline Road & S & SP & RSP & Road & S & SP & RSP \\
\hline 1 & B & A & B & 36 & C & C & B \\
\hline 4 & D & D & C & 37 & C & C & B \\
\hline 13 & B & A & B & 40 & B & A & B \\
\hline 14 & B & A & B & 41 & B & A & A \\
\hline 15 & B & A & B & 45 & B & A & B \\
\hline 16 & B & A & A & 46 & B & A & A \\
\hline 18 & B & A & B & 47 & C & B & A \\
\hline 19 & B & A & B & 48 & B & A & C \\
\hline 23 & C & B & B & 55 & C & C & B \\
\hline 29 & C & C & B & 58 & C & B & A \\
\hline 34 & C & B & B & 79 & C & B & A \\
\hline
\end{tabular}

Where, S is the standard method, SP is the set pair analysis, RSP is the revised set pair analysis. Among them, the grey square indicates that the evaluation grade obtained by normative method is lower than that of the set pair analysis, and the black square indicates that the evaluation grade obtained by the standard method is higher than that of the set pair analysis.

\subsection{Analysis and Discussion}

\subsubsection{Overall Road Sections Evaluation Analysis}

We found that when used the three methods to evaluate the pavement performance of 85 sections, the results would be different. This paper will divide all the results into seven categories for discussion.

The standard evaluation and set pair analysis evaluation comparison. 24 sections rose from level B to level $\mathrm{A}$ and 9 sections rose from level $\mathrm{C}$ to level B.

The standard evaluation and revised set pair analysis evaluation comparison. 23 sections rose, of which 3 sections rose directly from level $\mathrm{C}$ to level A and only 1 section fell from level B to level C.

The set pair analysis evaluation and revised set pair analysis evaluation comparison. Among them, the level of 10 sections increased and the level of 19 sections decreased.

The standard evaluation, set pair analysis evaluation and revised set pair analysis evaluation three methods are different. A total of 4 sections, one of the traditional specification is level $\mathrm{B}$, set pair analysis is level A, modified set pair analysis method is level C.

The set pair analysis evaluation and revised set pair analysis evaluation are the same, but different from the standard evaluation. There are 12 sections, and the evaluation level is higher than the standard.

The set pair analysis evaluation and standard evaluation are the same, but different from revised set pair analysis evaluation. There are 8 sections, and the evaluation level is lower than the revised set pair analysis.

The standard evaluation and revised set pair analysis evaluation are the same, but different from set pair analysis evaluation. A total of 17 sections and the evaluation level is lower than the set pair analysis.

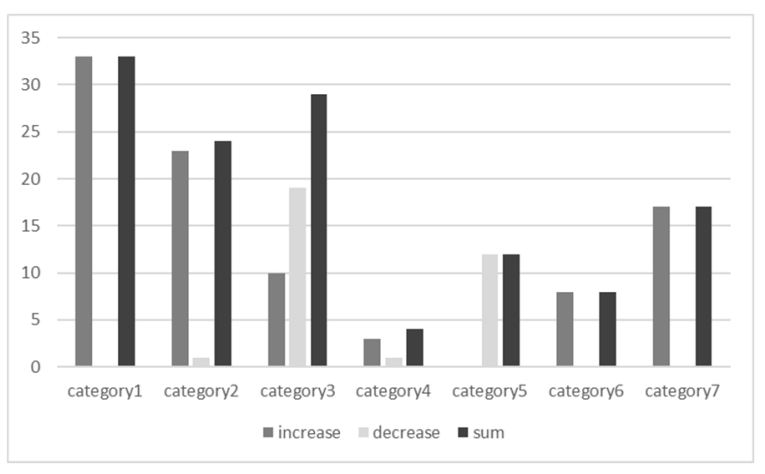

Figure 1 Statistical chart of seven road grades

Combined with the actual road conditions, the road has undergone an overall overhaul shortly before the detection period, so it is in line with the pavement performance at a good level. The rating of the standard method is lower than that of set pair analysis and revised set pair analysis, indicating that the predicted pavement life is the shortest. Set pair analysis has the best overall 
rating, indicating that the predicted pavement life is the longest. The revised set pair analysis is evaluated in both, and the method is reasonable. It not only avoids the waste of material resources and high economic cost caused by too short prediction, but also avoids the potential safety hazard caused by too long prediction and poor road condition to traffic users.

\subsubsection{Specific Road Sections Evaluation Analysis}

Among the evaluation grades obtained by the three evaluation methods, it is found that one kind of situation is special: the grades of three methods are different with each other. To further analyze the reasons, we selected four roads for specific analysis to prove the rationality of the revised set pair analysis.

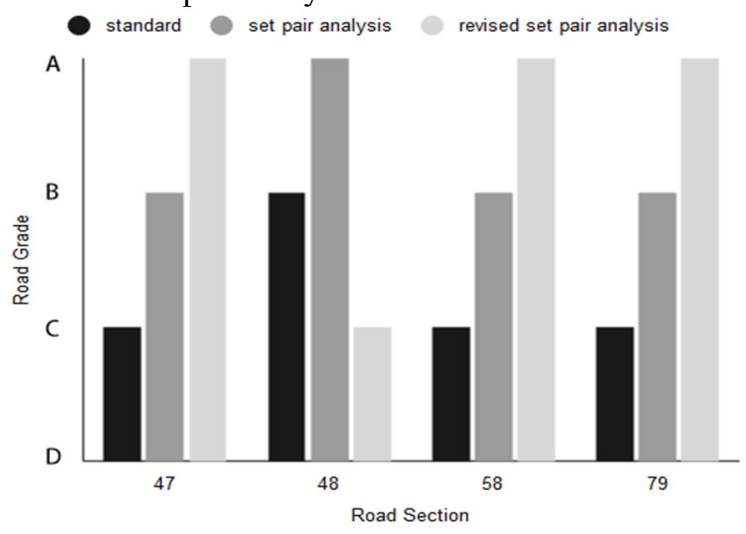

Figure 2 Evaluation grade comparison chart

Figure 2 shows that the grades obtained by three methods of the four sections are different. Among the sections 47,48 and 58, the revised set pair analysis has the highest level, followed by the set pair analysis and the standard method. Section 48 set pair analysis level is the highest, revised set pair analysis level is the lowest.

Through the actual investigation, there is only partial line crack in the damaged area of section 47 , and other damages are minimal. However, the calculated PCI is 78.7, which is inconsistent with the actual situation. Compared with the $\mathrm{C}$ level obtained by the standard method, level $\mathrm{A}$ is more in line with the actual road conditions. The RQI calculated by sections 48 and 79 are 3.03 and 3.25 , respectively, with excellent flatness. In addition, there are no other damage types except line cracks and mesh cracks. The overall driving comfort is well, and grade A is more realistic. Section 48 has a high level of set pair analysis, and the revised set pair analysis is lower than the traditional one. Although the RQI is 3.76 and the flatness is well, but in the set pair analysis to judge the influence degree, the RQI coefficient is 0.118 , PCI is 0.818 . Obviously, the influence degree of PCI is much larger than that of RQI. The subsidence area of this road section is 3.61 while the pit area is 2.0. The actual driving is not smooth and the comfort is poor, so the $\mathrm{C}$ level can better reflect the actual road grade of the road section.

To sum up, in the evaluation and analysis process of specific road sections, combined with the actual road sections such as road roughness and damaged area, the revised set pair analysis can better reflect the actual road condition level of the road.

\section{Conclusion}

Based on the existing research, the paper selects road sections in Shanghai as the research object, and conducts a comprehensive investigation on the pavement condition. In addition, we compare the advantages and disadvantages of various survey methods, and select the best.

Through the method comparison and field research, the comprehensive evaluation results and the subevaluation results are contradictory when the standard evaluation method evaluates the individual sections. The set pair analysis has the problem of inaccurate evaluation due to the large difference of sub-indexes. The revised set pair analysis can better solve the evaluation defects of the standard evaluation and the equidistant set pair analysis before revision, and more accurately reflect the actual pavement performance.

However, the current data selection is limited to urban roads, and the research on highways remains to be considered. Later teams will investigate more roads to verify the rationality of the method and propose maintenance plans for different levels of roads.

\section{Acknowledgements}

This work was supported by a grant from Sponsored by Shanghai Sailing Program (20YF1431900).

I would like to show my deepest gratitude to my supervisor, Dr. Sun Yu, who has provided me with valuable guidance in every stage. Then, I pleased to acknowledge my classmates Xu H, Zhu QY, for their invaluable assistance throughout the preparation of the original manuscript.

\section{References}

1. AASHTO, AASHTO Guidelines For Pavement Management Systems[S].America Association of State Highway and Transportation Officials , Washington D.C, 1990

2. Maria, T., M. Amelia, and S. Vembrie. "Evaluation of Toll Road Pavement Performance Based on the 2013 Bina Marga Method (Case Study: Serpong-Pondok Aren Toll Road)." IOP Conference Series: Earth and Environmental Science. Vol. 498. No. 1. IOP Publishing, 2020.

3. Murata, Yoshinobu, et al. "Long-life repair method for road based on soundness evaluation of embankment and pavement." Japanese Geotechnical Society Special Publication 8.11 (2020): 424-429.

4. Majidifard, Hamed, et al. "Developing a prediction model for rutting depth of asphalt mixtures using gene expression programming." Construction and Building Materials 267 (2020): 120543.

5. Fan W, Dai L.Pavement evaluation with AHP based on expert's assessment[C]//2015 23rd International 
Conference on Geoinformatics.IEEE,2015.

6. Yang, Sanqiang, et al. "Highway Performance Evaluation Index in Semiarid Climate Region Based on Fuzzy Mathematics." Advances in Materials Science and Engineering 2019 (2019).

7. Fan, Xu-ying, et al. "Establishment of an evaluation model for asphalt pavement preventive maintenance based on improved EW-AHP." Journal of Highway and Transportation Research and Denelopment 11.3 (2017): 48-53. 